\title{
Does the leaf economic spectrum hold within plant functional types? A Bayesian multivariate trait meta-analysis
}

\author{
Alexey N. Shiklomanov iD ${ }^{1,12}$ Elizabeth M. Cowdery, ${ }^{2}$ Michael Bahn, ${ }^{3}$ Chaeho Byun, ${ }^{4}$ Steven Jansen, ${ }^{5}$ \\ Koen Kramer, ${ }^{6}$ Vanessa Minden, ${ }^{7,8}$ Ülo Ninnemets, ${ }^{9}$ Yusuke Onoda, ${ }^{10}$ Nadejda A. Soudzilovskaia, ${ }^{11}$ And \\ Michael C. Dietze (iD ${ }^{2}$ \\ ${ }^{1}$ Joint Global Change Research Institute, Pacific Northwest National Laboratory, College Park, Maryland 20740 USA \\ ${ }^{2}$ Department of Earth \& Environment, Boston University, 685 Commonwealth Avenue Boston, Massachusetts 02215 USA \\ ${ }^{3}$ Institute of Ecology, University of Innsbruck, Innsbruck 6020 Austria \\ ${ }^{4}$ School of Civil and Environmental Engineering, Yonsei University, Seoul 03722 Korea \\ ${ }^{5}$ Institute of Systematic Botany and Ecology, Ulm University, Albert-Einstein-Allee 11, Ulm 89081 Germany \\ ${ }^{6}$ Department of Vegetation, Forest, and Landscape Ecology, Wageningen Environmental Research and Wageningen University, \\ P.O. Box 6708, Droevendaalsesteeg 4, Wageningen The Netherlands \\ ${ }^{7}$ Institute for Biology and Environmental Sciences, Carl von Ossietzky-University of Oldenburg, Carl von Ossietzky Strasse 9-11, \\ Oldenburg 26129 Germany \\ ${ }^{8}$ Department of Biology, Ecology and Evolution, Vrije Universiteit Brussel, Pleinlaan 2, Brussels 1050 Belgium \\ ${ }^{9}$ Institute of Agricultural and Environmental Sciences, Estonian University of Life Sciences, Kreutzwaldi 1, Tartu 51014 Estonia \\ ${ }^{10}$ Graduate School of Agriculture, Kyoto University, Kyoto 605-8503 Japan \\ ${ }^{11}$ Conservation Biology Department, Institute of Environmental Sciences, Leiden University, Rapenburg 70, 2311 EZ Leiden,
} The Netherlands

Citation: Shiklomanov, A. N., E. M. Cowdery, M. Bahn, C. Byun, S. Jansen, K. Kramer, V. Minden, Ü. Niinemets, Y. Onoda, N. A. Soudzilovskaia, and M. C. Dietze. 2020. Does the leaf economic spectrum hold within plant functional types? A Bayesian multivariate trait meta-analysis. Ecological Applications 30(3):e02064. 10.1002/eap.2064

Abstract. The leaf economic spectrum is a widely studied axis of plant trait variability that defines a trade-off between leaf longevity and productivity. While this has been investigated at the global scale, where it is robust, and at local scales, where deviations from it are common, it has received less attention at the intermediate scale of plant functional types (PFTs). We investigated whether global leaf economic relationships are also present within the scale of plant functional types (PFTs) commonly used by Earth System models, and the extent to which this global-PFT hierarchy can be used to constrain trait estimates. We developed a hierarchical multivariate Bayesian model that assumes separate means and covariance structures within and across PFTs and fit this model to seven leaf traits from the TRY database related to leaf longevity, morphology, biochemistry, and photosynthetic metabolism. Although patterns of trait covariation were generally consistent with the leaf economic spectrum, we found three approximate tiers to this consistency. Relationships among morphological and biochemical traits (specific leaf area [SLA], N, P) were the most robust within and across PFTs, suggesting that covariation in these traits is driven by universal leaf construction trade-offs and stoichiometry. Relationships among metabolic traits (dark respiration $\left[R_{\mathrm{d}}\right]$, maximum $\mathrm{RuBisCo}$ carboxylation rate $\left[V_{\mathrm{c}, \max }\right]$, maximum electron transport rate $\left[J_{\max }\right]$ ) were slightly less consistent, reflecting in part their much sparser sampling (especially for high-latitude PFTs), but also pointing to more flexible plasticity in plant metabolistm. Finally, relationships involving leaf lifespan were the least consistent, indicating that leaf economic relationships related to leaf lifespan are dominated by across-PFT differences and that within-PFT variation in leaf lifespan is more complex and idiosyncratic. Across all traits, this covariance was an important source of information, as evidenced by the improved imputation accuracy and reduced predictive uncertainty in multivariate models compared to univariate models. Ultimately, our study reaffirms the value of studying not just individual traits but the multivariate trait space and the utility of hierarchical modeling for studying the scale dependence of trait relationships.

Key words: ecological modeling; functional trade-off; hierarchical modeling; leaf biochemistry; leaf morphology; trait variation.

\section{INTRODUCTION}

Manuscript received 1 April 2019; revised 1 November 2019; accepted 13 November 2019. Corresponding Editor: David S. Schimel.

${ }^{12}$ E-mail: alexey.shiklomanov@pnnl.gov
Plant functional traits link directly measurable features of individuals to their fitness within an ecosystem, and are often related to various aspects of whole-ecosystem function (Violle et al. 2007, Cardinale 
et al. 2012). Although global trait databases are larger and more open now than ever before, large gaps and sampling biases in these databases continue to pose a challenge to trait ecology (Cornwell et al. 2019). If all traits and plant species were completely independent from each other, the only way forward would be to collect more trait data, which is expensive and time consuming (Cornwell et al. 2019). Fortunately, recent trait syntheses have revealed that variability in plant functional traits is constrained by biophysical limitations and trade-offs between ecological strategies (Wright et al. 2004, Kattge et al. 2011, Díaz et al. 2015, Kleyer and Minden 2015).

One such constraint is the "leaf economic spectrum," which defines a negative relationship between specific leaf area (SLA) and leaf lifespan, and a positive relationship of SLA with $\mathrm{N}_{\text {mass }}, \mathrm{P}_{\text {mass }}$, and photosynthesis and respiration rates (Wright et al. 2004, Shipley et al. 2006, Reich 2014, Díaz et al. 2015). Leaf economic traits are correlated with plant productivity (Shipley et al. 2005, Niinemets 2016, Wu et al. 2016b), litter decomposition rates (Bakker et al. 2010, Hobbie 2015), community composition (Burns 2004, Cavender-Bares et al. 2004), and ecosystem function (Diaz et al. 2004, Musavi et al. 2015). The position of species along the leaf economic spectrum is related to climate and soil conditions (Wright et al. 2004, 2005, Cornwell and Ackerly 2009, Ordoñez et al. 2009, Wigley et al. 2016). As a result, relationships between leaf economic traits and climate have been used in ecosystem models to more finely resolve variation in plant function (Scheiter et al. 2013, Sakschewski et al. 2015, Verheijen et al. 2015).

The global, interspecific trait space in which the classic leaf economic spectrum was defined is the end result of a multitude of different processes operating at different spatial, temporal, and phylogenetic scales. The subset of these processes operating on time scales of centuries to millennia, such as evolution or turnover in soil carbon and nutrients, may not be relevant for predicting how individual plants and ecosystems will respond to changes on policy-relevant timescales of months to decades (Shaw and Etterson 2012). The extent to which processes operating on shorter timescales result in the same trait trade-offs is an open question in trait ecology. Observational studies of trait correlations at smaller spatial scales, such as sites, species, and individuals, produce inconsistent results, with some studies finding consistent correlations across scales (Wright et al. 2004, Albert et al. 2010a, Asner et al. 2014) and others that correlation strength and direction are scale dependent (Albert et al. 2010b, Messier et al. 2010, 2017a, Wright and Sutton-Grier 2012, Feng and Dietze 2013, Kichenin et al. 2013, Grubb et al. 2015, Wigley et al. 2016).

Many mechanisms have been suggested for scale dependence of trait relationships. Trade-offs may only apply when multiple competing strategies co-occur, and alternative processes can drive community assembly where strong environmental filters severely limit the range of feasible strategies (Grime and Pierce 2012, Rosado and de Mattos 2017). Different selective pressures dominate at different scales, particularly within vs. across species (Albert et al. 2010b, Messier et al. 2010, Kichenin et al. 2013), and different traits have different sensitivities to such pressures (Messier et al. 2017b). Experimental evidence shows that species can alter different aspects of their leaf economy independently (Wright and Sutton-Grier 2012). Global analyses show that allocation patterns (and therefore investment strategies and trait relationships) vary across plant functional types (Ghimire et al. 2017). Moreover, plants maintain their fitness through multiple strategies, not just leaf economics, which can lead to multiple mutually orthogonal axes of trait variability. As a result, changes in leaf economic traits often fail to predict changes in other aspects of plant function, such as hydraulics (Li et al. 2015), dispersal (Westoby et al. 2002), and overall plant carbon budget (Edwards et al. 2014).

For these reasons, observed global trait relationships may have limited predictive power at finer scales. On the other hand, trying to understand an ecosystem through bottom-up approaches starting with individual species is also challenging. For one, the required species-specific trait observations do not exist for a very large number of species (Cornwell et al. 2019). Even where sufficient trait data are available, scaling functional traits to ecosystem-scale processes also requires data on species relative abundance (Grime 1998), which can be even more uncertain than the trait data (Clark 2016). Finally, plant interactions can result in community-level responses to environmental change that are distinct from the sum of species-specific changes (Poorter and Navas 2003).

An intermediate strategy is to aggregate species with similar structural and functional characteristics into plant functional types (PFTs). Although PFTs are most widely used as the unit of plant functional differentiation in dynamic vegetation and earth system models (Prentice et al. 1992, Lavorel et al. 1997, Wullschleger et al. 2014), the underlying concept of plant assemblages has been an important part of ecological discourse for over a century (Cowles 1899, Clements 1936, Naeem and Wright 2003). Patterns of trait variation within and across PFTs are relevant for several reasons. First, trait covariance can be leveraged to impute missing trait values (Swenson 2013); the extent to which leveraging trait covariance reduces the size (i.e., variability and/or uncertainty) of the trait space has important implications for quantifying the parametric uncertainty in vegetation model projections (Dietze et al. 2013, 2014, LeBauer et al. 2013). Second, the large uncertainty in model projections of future global carbon budgets (Friedlingstein et al. 2006, 2014) has been at least partially attributed to the models' failure to account for plant adaptation to changing environments (Sitch et al. 2008), which has led to an increased interest in adding within-PFT trait 
plasticity to models (Van Bodegom et al. 2011, Verheijen et al. 2015).

While the leaf economic spectrum has been investigated at the global scale, where it is robust, and at local scales, where deviations from it are common, it has received less attention at the intermediate scale of PFTs. Thus, this paper seeks to answer the following questions: First, does the leaf economic spectrum hold within vs. across PFTs? Second, can the leaf economic spectrum and similar covariance patterns be leveraged to reduce uncertainties in trait estimates, particularly under data limitation? The answers to these question have implications for both functional ecology and ecosystem modeling. To address these questions, we developed a hierarchical multivariate Bayesian model that explicitly accounts for across- and within-PFT variability in trait correlations. We then fit this model to a global trait database to estimate mean trait values and variance-covariance matrices for PFTs as defined in a major earth system model (Community Land Model, CLM; Oleson et al. 2013). We evaluate the ability of this model to reduce uncertainties in trait estimates and reproduce observed patterns of global trait variation compared to univariate models. Finally, we assess the scale dependence and generality of estimated trait covariances.

\section{Materials And Methods}

\section{Trait data}

We focused on seven leaf traits obtained from the TRY global database (Kattge et al. 2011; Appendix S1): longevity (months), specific leaf area (SLA, $\mathrm{m}^{2} / \mathrm{kg}$ ), nitrogen content $\left(\mathrm{N}_{\text {mass }}, \mathrm{mg} \mathrm{N} / \mathrm{g}\right.$ or $\left.\mathrm{N}_{\text {area }}, \mathrm{g} / \mathrm{m}^{2}\right)$, phosphorus content $\left(\mathrm{P}_{\text {mass }}, \mathrm{mg} \mathrm{P} / \mathrm{g}\right.$ or $\left.\mathrm{P}_{\text {area }}, \mathrm{g} / \mathrm{m}^{2}\right)$, dark respiration at $25^{\circ} \mathrm{C} \quad\left(R_{\mathrm{d} \text {,mass }} \mu \mathrm{mol} \cdot \mathrm{g}^{-1} \cdot \mathrm{s}^{-1}\right.$, or $R_{\mathrm{d} \text {,area }}$, $\left.\mu \mathrm{mol} \cdot \mathrm{m}^{-2} \cdot \mathrm{s}^{-1}\right)$, maximum RuBisCO carboxylation rate at $25^{\circ} \mathrm{C} \quad\left(V_{\mathrm{c}, \text { max,mass, }} \mu \mathrm{mol} \cdot \mathrm{g}^{-1} \cdot \mathrm{s}^{-1}\right.$, or $V_{\mathrm{c}, \text { max,area }}$, $\left.\mu \mathrm{mol} \cdot \mathrm{m}^{-2} \cdot \mathrm{s}^{-1}\right)$, and maximum electron transport rate at $25^{\circ} \mathrm{C}\left(J_{\text {max,mass, }} \mu \mathrm{mol} \cdot \mathrm{g}^{-1} \cdot \mathrm{s}^{-1}\right.$, or $J_{\text {max,area }}$, $\left.\mu \mathrm{mol} \cdot \mathrm{m}^{-2} \cdot \mathrm{s}^{-1}\right)$. For $V_{\mathrm{c}, \max }$, we only used values reported at $25^{\circ} \mathrm{C}$. For $R_{\mathrm{d}}$ and $J_{\max }$, we normalized the values to $25^{\circ} \mathrm{C}$ using reported leaf temperature values following Atkin et al. (2015) and Kattge and Knorr (2007: Eq. 1 therein), respectively. To avoid issues with trait normalization, we performed analyses separately for both massand area-normalized traits (Lloyd et al. 2013, Osnas et al. 2013). We restricted our analysis to qualitycontrolled values from species with sufficient information for functional type classification (Kattge et al. 2011). Following past studies (Wright et al. 2004, Onoda et al. 2011, Díaz et al. 2015), we log-transformed all trait values to correct for their strong right-skewness.

\section{Plant functional types}

We assigned each species a PFT following the scheme in the Community Land Model (CLM4.5, Oleson et al.
2013; Table 1, Fig. 1). We obtained categorical data on growth form, leaf type, phenology, and photosynthetic pathway from TRY. Where species attributes disagreed between data sets, we assigned the most frequently observed attribute (e.g., if five data sets say "shrub" but only one says "tree," we would use "shrub"). Where species attributes were missing, we assigned attributes based on higher order phylogeny if possible (e.g., Poaceae family are grasses, Larix genus are deciduous needleleaved trees) or omitted the species if not. For biome specification, we matched geographic coordinates for each species to annual mean temperature (AMT, averaged 1970-2000) data from WorldClim-2 (Fick and Hijmans 2017), calculated the mean AMT for all sites where each species was observed, and then binned these species based on the following cutoffs: boreal/arctic $\left(\mathrm{AMT} \leq 5^{\circ} \mathrm{C}\right)$, temperate $\left(\mathrm{AMT} \leq 20^{\circ} \mathrm{C}\right)$, and tropical $\left(\mathrm{AMT}>20^{\circ} \mathrm{C}\right)$.

\section{Multivariate analysis}

Basic model description. - We compared three models with different levels of complexity. The simplest was the "univariate" model, in which each trait is independent. For an observation $x_{i, t}$ of trait $t$ and sample $i$

$$
x_{i, t} \sim N\left(\mu_{t}, \sigma_{t}\right)
$$

where $N$ is the univariate Gaussian distribution with mean $\mu_{t}$ and standard deviation $\sigma_{t}$ for trait $t$.

The second-simplest model was the "multivariate" model, in which traits are drawn from a single multivariate distribution. For observed trait vector $\mathbf{x}_{i}$ for sample $i$

$$
\mathbf{x}_{i} \sim \operatorname{mvN}(\boldsymbol{\mu}, \boldsymbol{\Sigma})
$$

where $\mathrm{mvN}$ is the multivariate Gaussian distribution with mean vector $\boldsymbol{\mu}$ and covariance matrix $\boldsymbol{\Sigma}$. We fit both of these models independently for each PFT and once for the entire data set (i.e., one global PFT).

The most complex model was the "hierarchical multivariate" model (henceforth, just "hierarchical model"), where traits are drawn from a PFT-specific multivariate distribution describing within-PFT variation, and whose mean vector is itself sampled from a global multivariate distribution describing variation across PFTs. For observed trait vector $\mathbf{x}_{i, p}$ for sample $i$ belonging to $\operatorname{PFT} p$

$$
\begin{gathered}
\mathbf{x}_{i, p} \sim \operatorname{mvN}\left(\boldsymbol{\mu}_{p}, \boldsymbol{\Sigma}_{p}\right) \\
\boldsymbol{\mu}_{p} \sim \operatorname{mvN}\left(\boldsymbol{\mu}_{g}, \boldsymbol{\Sigma}_{g}\right)
\end{gathered}
$$

where $\boldsymbol{\mu}_{p}$ and $\boldsymbol{\Sigma}_{p}$ are the mean vector and covariance matrix describing variation within PFT $p$, and $\boldsymbol{\mu}_{g}$ and $\boldsymbol{\Sigma}_{g}$ are the mean vector and covariance matrix describing across-PFT (global) variation. 
TABLE 1. Names, labels, species counts, and number of non-missing observations of each trait for plant functional types (PFTs) used in this analysis.

\begin{tabular}{|c|c|c|c|c|c|c|c|c|c|c|c|c|c|c|}
\hline \multirow[b]{2}{*}{ Label } & \multirow[b]{2}{*}{ PFT } & \multirow[b]{2}{*}{ Species } & \multirow{2}{*}{$\begin{array}{c}\text { Leaf } \\
\text { lifespan }\end{array}$} & \multirow[b]{2}{*}{ SLA } & \multicolumn{5}{|c|}{ Mass } & \multicolumn{5}{|c|}{ Area } \\
\hline & & & & & $\mathrm{N}$ & $\mathrm{P}$ & $R_{\mathrm{d}}$ & $V_{\mathrm{c}, \max }$ & $J_{\max }$ & $\mathrm{N}$ & $\mathrm{P}$ & $R_{\mathrm{d}}$ & $V_{\mathrm{c}, \max }$ & $J_{\max }$ \\
\hline BlETr & $\begin{array}{l}\text { Broadleaf } \\
\text { evergreen } \\
\text { tropical tree }\end{array}$ & 1,229 & 153 & 11,710 & 7,547 & 2,912 & 237 & 205 & 58 & 4,023 & 1,684 & 326 & 225 & 152 \\
\hline BlETe & $\begin{array}{l}\text { Broadleaf } \\
\text { evergreen } \\
\text { temperate tree }\end{array}$ & 363 & 135 & 2,210 & 1,811 & 1,194 & 121 & 36 & 16 & 928 & 339 & 196 & 106 & 87 \\
\hline BlDTr & $\begin{array}{l}\text { Broadleaf } \\
\text { deciduous } \\
\text { tropical tree }\end{array}$ & 286 & 82 & 2,166 & 1,545 & 812 & 98 & 54 & 30 & 813 & 500 & 113 & 56 & 53 \\
\hline BlDTe & $\begin{array}{l}\text { Broadleaf } \\
\text { deciduous } \\
\text { temperate tree }\end{array}$ & 345 & 181 & 9,536 & 5,982 & 2,163 & 942 & 245 & 576 & 2,163 & 398 & 866 & 697 & 849 \\
\hline BIDBo & $\begin{array}{l}\text { Broadleaf } \\
\text { deciduous } \\
\text { boreal tree }\end{array}$ & 62 & 58 & 908 & 898 & 340 & 142 & 0 & 0 & 141 & 60 & 11 & 5 & 5 \\
\hline NlETe & $\begin{array}{l}\text { Needleleaf } \\
\text { evergreen } \\
\text { temperate tree }\end{array}$ & 130 & 66 & 2,958 & 4,940 & 3,729 & 262 & 92 & 91 & 1,227 & 462 & 84 & 274 & 106 \\
\hline NlEBo & $\begin{array}{c}\text { Needleleaf } \\
\text { evergreen } \\
\text { boreal tree }\end{array}$ & 30 & 24 & 530 & 1,457 & 393 & 493 & 0 & 0 & 101 & 14 & 16 & 3 & 3 \\
\hline NID & $\begin{array}{l}\text { Needleleaf } \\
\text { deciduous tree }\end{array}$ & 19 & 16 & 195 & 328 & 179 & 34 & 1 & 0 & 48 & 10 & 3 & 4 & 0 \\
\hline ShE & Shrub evergreen & 1,120 & 298 & 5,018 & 3,555 & 2,404 & 207 & 22 & 13 & 1,376 & 747 & 205 & 41 & 32 \\
\hline ShDTe & $\begin{array}{l}\text { Shrub deciduous } \\
\text { temperate }\end{array}$ & 330 & 100 & 3,026 & 1,525 & 1,227 & 10 & 9 & 1 & 576 & 281 & 13 & 33 & 19 \\
\hline ShDBo & $\begin{array}{l}\text { Shrub deciduous } \\
\text { boreal }\end{array}$ & 94 & 80 & 482 & 552 & 313 & 0 & 1 & 1 & 133 & 51 & 0 & 1 & 1 \\
\hline C3GAr & $\mathrm{C}_{3}$ grass arctic & 157 & 65 & 989 & 996 & 573 & 11 & 1 & 2 & 219 & 85 & 7 & 1 & 2 \\
\hline $\mathrm{C} 3 \mathrm{GTe}$ & $\begin{array}{l}\mathrm{C}_{3} \text { grass } \\
\text { temperate }\end{array}$ & 624 & 76 & 6,322 & 3,802 & 1,541 & 103 & 21 & 27 & 1,257 & 382 & 93 & 52 & 47 \\
\hline $\mathrm{C} 4 \mathrm{G}$ & $\mathrm{C}_{4}$ grass & 255 & 31 & 1,312 & 1,461 & 335 & 44 & 0 & 0 & 410 & 56 & 28 & 0 & 0 \\
\hline
\end{tabular}

Notes: SLA, specific leaf area; $R_{\mathrm{d}}$, dark respiration; $V_{\mathrm{c}, \max }$, maximum RuBisCo carboxylation rate at $25^{\circ} \mathrm{C} ; J_{\max }$, maximum electron transport rate at $25^{\circ} \mathrm{C}$.

\section{Model implementation}

We fit the above models using Gibbs sampling, which leverages conjugate prior relationships for efficient exploration of the sampling space. The main advantages of Gibbs sampling over distribution-agnostic Bayesian algorithms such as Metropolis Hastings (Haario et al. 2001), Differential Evolution (ter Braak and Vrugt 2008), and Hamiltonian Monte-Carlo (Neal 2011) is that Gibbs sampling has a $100 \%$ proposal acceptance rate (compared to $10-65 \%$ for these algorithms), meaning that it requires roughly 2-10 times fewer Markov Chain Monte Carlo (MCMC) iterations.

For priors on all multivariate mean vectors $(\boldsymbol{\mu})$, we used multivariate normal distributions. For priors on all multivariate variance-covariance matrices, we used the Wishart distribution $(W)$, which leads to the following posterior distribution

$$
P\left(\boldsymbol{\Sigma} \mid \mathbf{x}, \boldsymbol{\mu}, v_{0}, \mathbf{\Sigma}_{0}\right) \sim\left(W\left(v^{*}, S^{*}\right)\right)^{-1}
$$

$$
\begin{gathered}
v^{*}=1+v_{0}+n+m \\
\mathbf{S}^{*}=\left(\mathbf{S}_{0}+(\overline{\mathbf{x}}-\mu)^{T}(\overline{\mathbf{x}}-\mu)\right)^{-1}
\end{gathered}
$$

where $n$ is the number of observations, $m$ is the number of traits in data matrix $\mathbf{x}$, and $\overline{\mathbf{x}}$ is the column means of $\mathbf{x}$. For further details on the derivation of the conjugate relationship, see Gelman et al. (2003:72, Section 3.6).

We used weakly informative priors for trait means and variances (diagonals of the multivariate normal covariance matrix), the values of which are shown in Appendix S2: Table S1. All of the covariance (off-diagonal) terms in the prior variance matrix were set to zero. We used uninformative priors for the Wishart distribution $\left(v_{0}=0, \mathbf{S}_{0}=\operatorname{diag}(1, m)\right)$.

The above equations defining the conjugacy relationship do not work if the data matrix $\mathbf{x}$ has any missing values. Therefore, we modeled the partially missing observations as latent variables conditioned on the present observations and estimated mean vector and covariance 


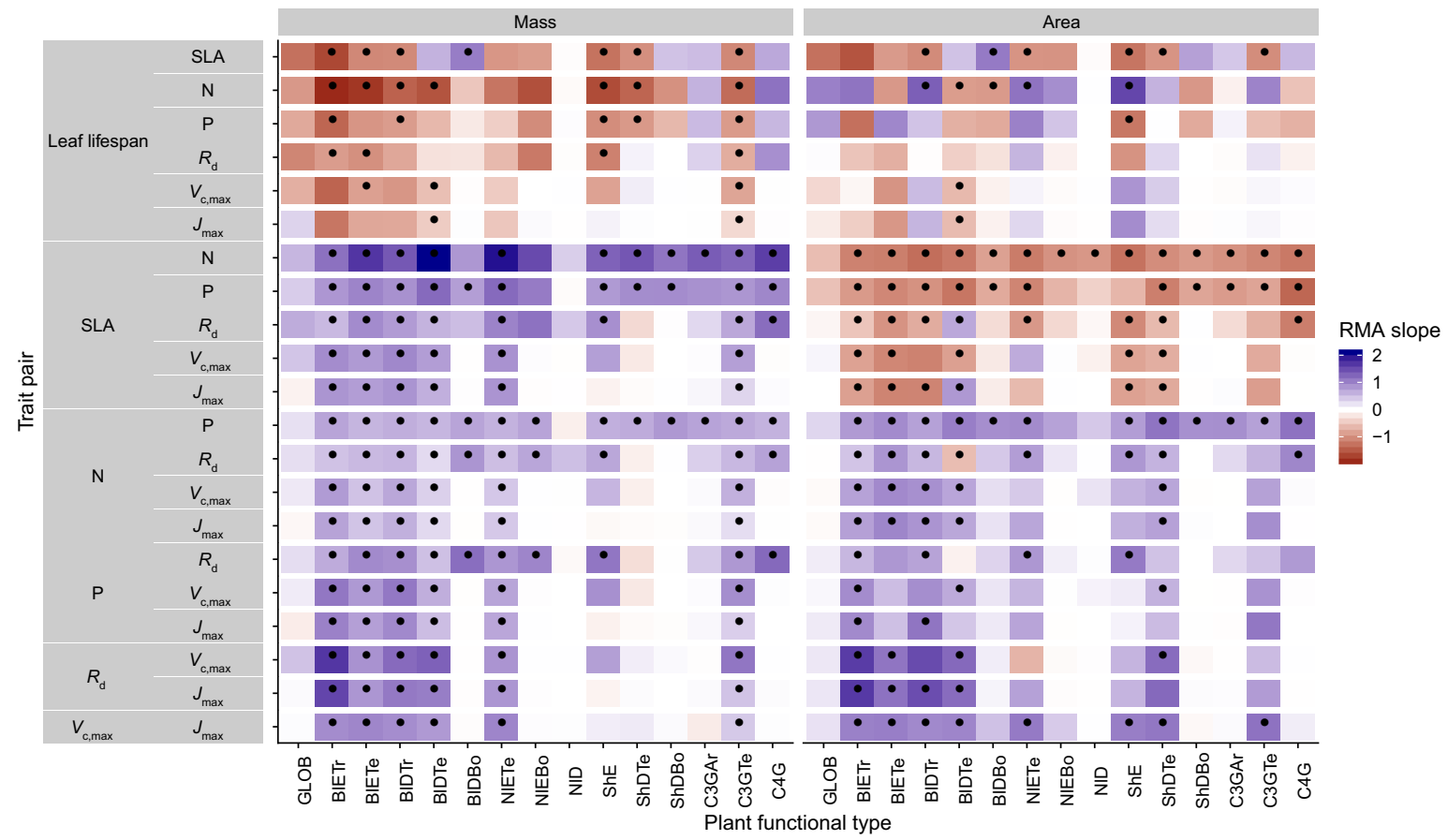

FIG. 1. Mean pairwise reduced major axis (RMA) slope estimates calculated from within- and across-PFT covariance matrix estimates from the hierarchical model. The slope numerator $(y)$ is the outer trait and the denominator $(x)$ is the inner trait (so in the top row, the slope is $\Delta$ (leaf lifespan)/ $\Delta$ (SLA)). Blue colors indicate positive slopes and red colors indicate negative slopes, with darker shades indicating steeper slopes. Points indicate slopes whose $95 \%$ credible intervals do not overlap zero. PFT, plant functional type; SLA, specific leaf area.

matrix. This approach is conceptually similar to multiple imputation (Graham 2009, White et al. 2010), and is quite distinct from single imputation, where data are imputed once in a separate step prior to parameter estimation (Graham 2009, White et al. 2010). For a block of data $\mathbf{x}^{\prime}$ containing missing observations in columns $\boldsymbol{m}$ and present observations in columns $\boldsymbol{p}$, missing values $\mathbf{x}^{\prime}[\mathrm{m}]$ are drawn randomly from a conditional multivariate normal distribution at each iteration of the sampling algorithm:

$$
\begin{gathered}
\mathbf{x}^{\prime}[m \mid p] \sim \operatorname{mvN}\left(\boldsymbol{\mu}^{\prime}, \boldsymbol{\Sigma}^{\prime}\right) \\
\boldsymbol{\mu}^{\prime}=\left(\mathbf{x}^{\prime}[p]-\boldsymbol{\mu}^{\prime}[p]\right)\left(\boldsymbol{\Sigma}[p, p]^{-1} \boldsymbol{\Sigma}[p, m]\right) \\
\boldsymbol{\Sigma}^{\prime}=\boldsymbol{\Sigma}[m, m]-\boldsymbol{\Sigma}[m, p]\left(\boldsymbol{\Sigma}[p, p]^{-1} \boldsymbol{\Sigma}[p, m]\right) .
\end{gathered}
$$

Sampling proceeds according to the following algorithm: Let $\boldsymbol{\mu}_{i}$ and $\boldsymbol{\Sigma}_{i}$ be the estimates of the mean vector and covariance matrix, respectively, at MCMC iteration $i$. Similarly, let $x_{i}$ be the realization of the data $\mathbf{x}^{\prime}$ with missing (latent) values imputed at MCMC iteration $i$.

1) Initialize $\mu_{1}$ and $\Sigma_{1}$ as a random draw from their respective priors.

2) Generate $x_{1}$ as a function of $\mu_{1}$ and $\Sigma_{1}$.

3) Draw $\mu_{2}$ and $\Sigma_{2}$ as a function of $x_{1}$, according to the corresponding Gibbs sampling step.
4) Generate $x_{2}$ as function of $\mu_{2}$ and $\boldsymbol{\Sigma}_{2}$.

5) Draw $\mu_{3}$ and $\Sigma_{3}$ as a function of $x_{2}$.

6) Continue alternating these steps until a stable distribution of $\mu$ and $\Sigma$ is reached.

A detailed demonstration of this approach is shown in Appendix S2: Section S1. By performing imputation at every MCMC iteration, we integrate over the uncertainty in the missing data. Combined with uninformative priors on the covariance centered on zero (as previously described), this means our approach provides an inherently conservative estimate of both trait covariances and imputed missing values. Where data are limited, our approach will tend towards covariance estimates of 0 with wide credible intervals, and the resulting weak and uninformative covariance estimates will lead to larger uncertainties in the imputed values.

For each model fit, we ran independent five chains, continuing sampling until the final result achieved convergence as determined by a univariate GelmanRubin potential scale reduction statistic less than 1.1 for all parameters (Gelman and Rubin 1992). We implemented this sampling algorithm in a publicly available R (R Core Team 2019) package (available online). ${ }^{13}$

\footnotetext{
${ }^{13} \mathrm{http} / / /$ github.com/ashiklom/mvtraits
} 


\section{Analysis of results}

To assess the consistency of within- and across-PFT trait trade-offs, we calculated the mean and $95 \%$ credible interval of the pairwise reduced major axis slope $(M)$ for each trait pair $(i, j)$ from posterior samples of their variancecovariance matrices $(\boldsymbol{\Sigma})$ using the following equation:

$$
M_{i, j}=\frac{\boldsymbol{\Sigma}_{j, j}}{\boldsymbol{\Sigma}_{i, i}} \operatorname{sign}\left(\boldsymbol{\Sigma}_{i, j}\right)
$$

Although this is a Bayesian analysis and therefore has no formal tests of statistical significance, we approximated the statistical significance of slope estimates as those whose $95 \%$ credible interval did not overlap zero. We calculated reduced major axis slopes both within and across PFTs.

To explore patterns of trait variation across PFTs, and to provide updated parameter values for earth system models, we calculated the mean and 95\% credible intervals of PFT-level trait estimates from our hierarchical model. We also compare these values to the default parameter values of CLM 4.5 (Oleson et al. 2013: Table 8.1) for SLA, $\mathrm{N}_{\text {mass }}, \mathrm{N}_{\text {area }}, V_{\mathrm{c} \text {,max,mass }}$ and $V_{\mathrm{c}, \max }$, area. To convert CLM's reported C: $\mathrm{N}$ ratio to $\mathrm{N}_{\text {mass, }}$, we assumed a uniform leaf $\mathrm{C}$ fraction of 0.46 . We then divided this calculated $\mathrm{N}_{\text {mass }}$ by the reported SLA to obtain $\mathrm{N}_{\text {area }}$. We calculated $V_{\mathrm{c} \text {,max,mass }}$ by multiplying the reported $V_{\mathrm{c} \text {,max,area }}$ by the reported SLA.

To compare the ability of the different models to predict missing trait observations, we performed a crossvalidation where we randomly removed 1,000 observations from the data and evaluated the ability of the fitted models to impute these missing observations. We report the results of the normalized mean root mean square error (RMSE) of these predicted observations.

To test whether multivariate and hierarchical models offer relatively more utility at smaller sample sizes, we calculated the relative uncertainty $(\alpha)$ as a function of the mean $(\mu)$ and upper $\left(q_{0.975}\right)$ and lower $\left(q_{0.025}\right)$ confidence limits of trait estimates

$$
\alpha=\frac{q_{0.975}-q_{0.025}}{\mu} .
$$

We then fit a log-linear least-squares regression relating relative uncertainty to sample size $(n)$ for each model (univariate, multivariate, and hierarchical; Fig. 4)

$$
\log \alpha=b_{0}+b_{1} \log n
$$

If all three models performed equally well at all sample sizes, their respective slope and intercept coefficients would be statistically indistinguishable. Meanwhile, models that perform better should have a lower intercept $\left(b_{0}\right)$, indicating lower overall uncertainty, and a lower slope $\left(b_{1}\right)$, indicating reduced sensitivity of uncertainty $(\alpha)$ to sample size $(n)$.

\section{Data and code availability}

All $\mathrm{R}$ analyses were run using $\mathrm{R}$ version 3.6.1 ( $\mathrm{R}$ Core Team 2019). The $\mathrm{R}$ code and data for running these analyses is publicly available online via the Open Science Framework (see Data Availability). To comply with TRY intellectual property guidelines, the trait data used in this study have been "anonymized" such that they can only be identified to the PFT level (not the species level) as required to reproduce this analysis. The complete TRY data request used for this analysis has been archived online (see Data Availability).

\section{RESULTS}

\section{Trait covariance patterns within and across PFTs}

The direction and magnitude of pairwise trait relationships was quite variable within and across PFTs (Fig. 1). Broadly, this variability can be captured by breaking up the seven leaf traits considered in this analysis into three groups: morphology and biochemistry (SLA, N, P), metabolism $\left(R_{\mathrm{d}}, V_{\mathrm{c}, \max }, J_{\max }\right)$, and leaf lifespan.

Morphological and biochemical traits (SLA, N, P) showed the most robust and consistent mutual covariance of these three groups. SLA was positively related to $\mathrm{N}_{\text {mass }}$ and $\mathrm{P}_{\text {mass }}$, and negatively related to $\mathrm{N}_{\text {area }}$ and $\mathrm{P}_{\text {area }}$, both across PFTs and within all PFTs. The magnitude of the slopes between $\mathrm{N}$ and $\mathrm{P}$ (regardless of normalization), and of SLA with $\mathrm{N}_{\text {area }}$ and $\mathrm{P}_{\text {area }}$, were relatively constant within all PFTs, but the magnitude of the slopes of SLA with $\mathrm{N}_{\text {mass }}$ and $\mathrm{P}_{\text {mass }}$ were more variable. In particular, temperate tree species (BlETe, BlDTe, N1ETe) showed steeper SLA-N $\mathrm{N}_{\text {mass }}$ slopes (more variation in SLA relative to $\mathrm{N}_{\text {mass }}$ ) than most other PFTs.

Covariance among metabolic traits $\left(R_{\mathrm{d}}, V_{\mathrm{c}, \max }, J_{\max }\right)$ was slightly less robust. Pairwise relationships among metabolic traits were weaker across-PFTs than withinPFTs. Across-PFT relationships among metabolic traits were also weaker than across-PFT relationships among SLA, N, and P. Within PFTs, the relationship between $V_{\text {c,max }}$ and $J_{\max }$ (regardless of normalization) was largely consistent in magnitude and direction, while the relationship of $R_{\mathrm{d}}$ with both of these traits was more variable. Within-PFT relationships of metabolic traits with $\mathrm{N}$ and $\mathrm{P}$ were usually positive, and relationships with SLA were usually positive under mass normalization and negative under area normalization. Two PFTs had notable deviations from these patterns under area normalization: Broadleaved deciduous temperate (B1DTe) trees had opposite slopes for the SLA- $R_{\mathrm{d} \text {,area, }} \mathrm{SLA}-$ $J_{\text {max,area }}$, and $\mathrm{N}_{\text {area }}-R_{\mathrm{d} \text {,area, }}$, while needleleaved evergreen temperate trees (N1ETe) had opposite slopes for $R_{\mathrm{d}, \text { area }}{ }^{-}$ $V_{\text {c,max,area }}$ and SLA $-V_{\text {c,max,area }}$. Finally, an important feature of metabolic traits is the much larger number of near-zero pairwise slope estimates, which is driven by 
the relative paucity of observations (especially pairwise observations) of these traits for many PFTs.

Slopes of all of the above traits with leaf lifespan showed the most variability. Across-PFT relationships of leaf lifespan with other traits were, on average, stronger than across-PFT relationships among the other traits, especially for mass-normalized traits. Within-PFT relationships of leaf lifespan with mass normalized traits were most often positive, but varied systematically with leaf habit and biome. Namely, among deciduous PFTs, leaf lifespan-SLA and leaf lifespan- $\mathrm{N}_{\text {mass }}$ slopes were less positive or more negative in colder biomes than warmer ones $(\mathrm{BlETr}>\mathrm{BlETe}, \mathrm{BIDTr}>\mathrm{BIDTe}>\mathrm{BIDBo}$, ShDTe $>$ ShDBo, C3GTe $>$ C3GAr). Meanwhile, slopes of leaf lifespan with area-normalized traits were generally weaker and idiosyncratic.

An important caveat to these results is that many slopes, including all of the across-PFT slopes, had 95\% credible intervals that intersected zero-i.e., we are less than $95 \%$ confident in the direction of these slopes. This is primarily due to variations in the effective number of pairwise observations used to estimate the covariance matrix: the more pairwise observations are available, the smaller the minimum covariance that can be estimated with the same level of statistical power and confidence. For example, a power analysis of correlation coefficients ('pwr::pwr.r.test' in R; Champely 2018) showed that, with 14 plant functional types $(n=14)$, the smallest across-PFT correlation we would be able to estimate with $95 \%$ power $(\alpha=0.95)$ and confidence $(P=0.05)$ is 0.74 , so we can confidently say that all PFT correlation coefficients (different from, but closely related to slope) were smaller than that value. That being said, because all across-PFT slopes have the same sample size, we can reasonably expect differences in the mean strength of pairwise across-PFT trait relationships to be ecologically meaningful. The situation is more complex for PFT-level estimates, where sample size varies by multiple orders of magnitude by PFT and trait pair (Table 1; Appendix S2: Table S4). In particular, high-latitude PFTs (BIDBo, NIEBo, NID, ShDBo, and C3GAr) and metabolic traits $\left(R_{\mathrm{d}}, V_{\mathrm{c}, \max }, J_{\max }\right)$ stand out as having particularly low sample sizes.

\section{Estimates of PFT-level means}

Across-PFT patterns in SLA, $\mathrm{N}_{\text {mass }}, \mathrm{P}_{\text {mass }}$, and $R_{\mathrm{d} \text {,mass }}$ were similar, with the highest values in temperate broadleaved deciduous PFTs and the lowest values in evergreen PFTs (Fig. 2). However, none of these patterns was universal to all four traits. For example, tropical evergreen trees had relatively high $\mathrm{N}_{\text {mass }}$ and average SLA and $R_{\mathrm{d} \text {,mass, }}$, but among the lowest $\mathrm{P}_{\text {mass. }}$. Similarly, compared to grass PFTs, temperate and boreal shrubs had similar SLA but higher $\mathrm{N}_{\text {mass }}$ and $\mathrm{P}_{\text {mass. }}$. Patterns were different when these traits were normalized by area instead of mass. For example, needleleaf evergreen trees had relatively low $\mathrm{N}_{\text {mass }}$ and $\mathrm{P}_{\text {mass }}$ but relatively high
$\mathrm{N}_{\text {area }}$ and $\mathrm{P}_{\text {area }}$, while the opposite was true of deciduous temperate trees and shrubs.

A key application of this study was to provide datadriven parameter estimates for Earth System models. To this end, we compared our mean parameter estimates with corresponding default parameters in CLM 4.5 (Oleson et al. 2013; Fig. 2). Our SLA estimates were lower (non-overlapping 95\% credible interval) than CLM parameters for all PFTs except tropical broadleaved evergreen trees. Our $\mathrm{N}_{\text {mass }}$ estimates showed more across-PFT variability than CLM parameters, and only agreed with CLM for evergreen temperate trees, needleleaved trees, and $\mathrm{C}_{3}$ Arctic grasses. Similarly to Kattge et al. (2009), we found that CLM overestimates $V_{\mathrm{c}, \max }$, both by mass and area.

\section{Comparing different models}

Both our multivariate and hierarchical models consistently outperformed the univariate approach in terms of their ability to impute missing trait values (Fig. 3). The relative amount of improvement from the univariate to the multivariate or hierarchical model was roughly proportional to the sample size of the underlying trait. For instance, for SLA, the best-sampled trait in our analysis, the hierarchical model's RMSE improved on the univariate model by only $4-6 \%$, while the improvement for the much more sparsely observed $V_{\mathrm{c}, \max }$ and $J_{\max }$ was $30-40 \%$. The differences between the grouped multivariate model and the hierarchical model were negligible, indicating that the additional information content of the across-PFT covariance is limited.

In general, leaf trait estimates from the univariate, multivariate, and hierarchical models were similar (Appendix S2: Fig. S1). Where estimates differed between models, the largest differences were between the univariate and multivariate models, and additional constraint from the hierarchical model relative to PFTspecific multivariate models had a minimal effect on trait estimates. Significant differences in trait estimates between univariate and multivariate models occurred even for well-sampled traits, such as leaf nitrogen content. We also observed differences in posterior predictive uncertainties of mean estimates with respect to sample size. High-latitude PFTs had large uncertainties relative to other PFTs, and the traits with the largest uncertainties were dark respiration, $V_{\mathrm{c}, \max }$, and $J_{\max }$. For many of these trait-PFT combinations, the additional constraint from trait covariance provided by the multivariate and hierarchical models reduced error bars, making it possible to compare estimates against those of other PFTs. Our analysis of the relationship between sample size and trait uncertainty found that, compared to the univariate model, the multivariate model both reduced uncertainty overall (lower intercept) and reduced the sensitivity of uncertainty to sample size (lower slope; Fig. 4). The hierarchical model further reduced both sensitivity to sample size and overall 

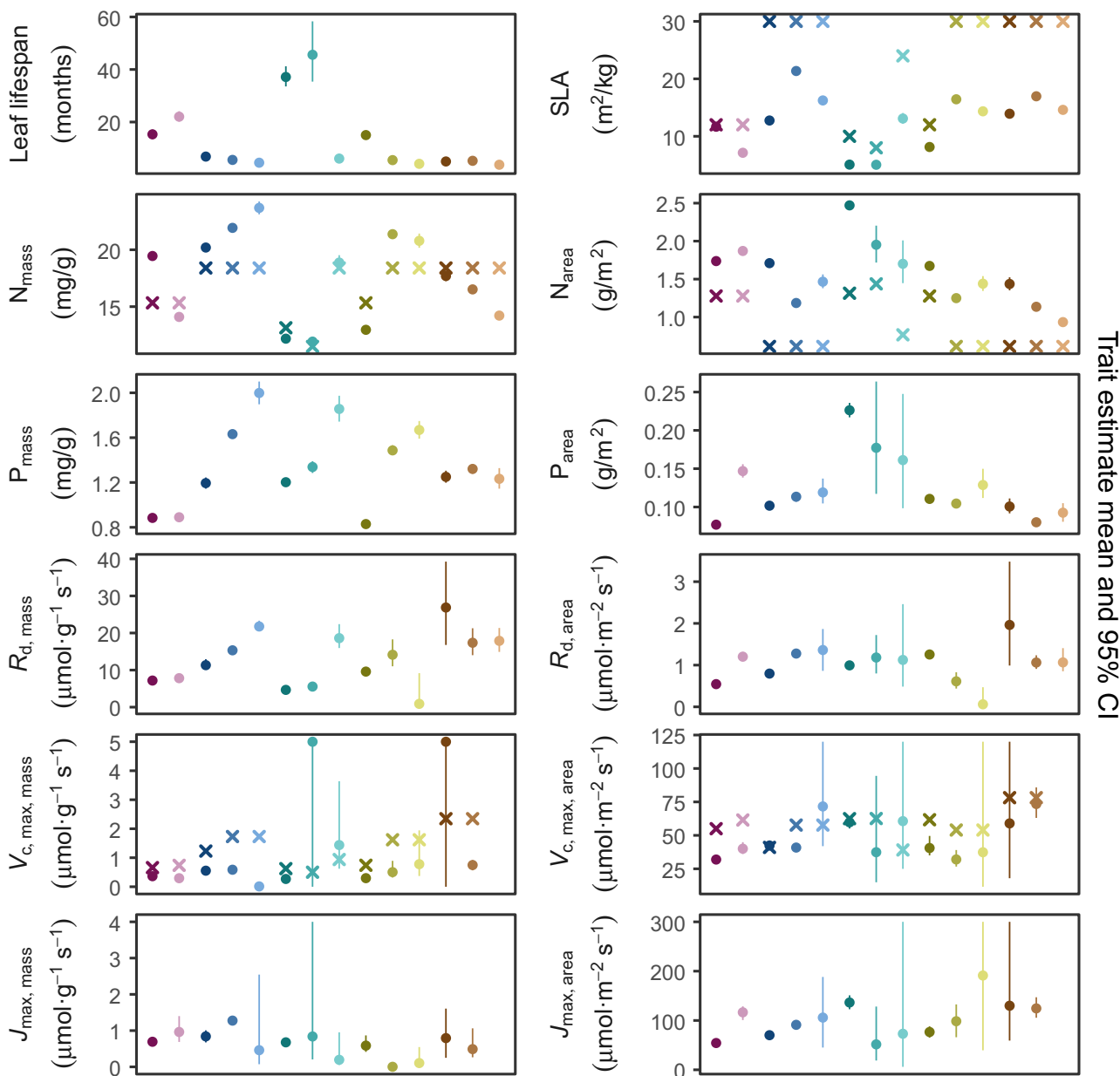

Plant functional type

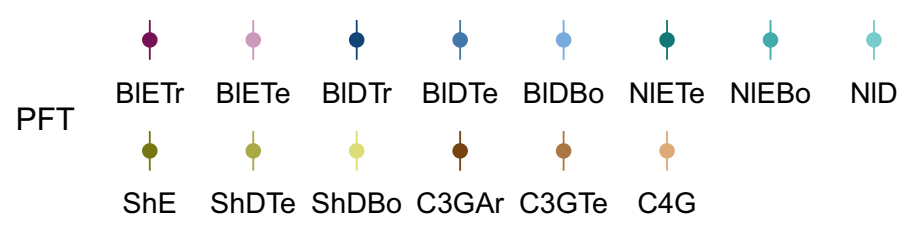

Model type

- hierarchical

$\times \quad$ CLM 4.5

FIG. 2. Mean and $95 \%$ credible interval on best estimates of traits for each plant functional type from the hierarchical model. For leaf lifespan and SLA, results were similar whether the other traits were normalized by mass or area, so only results from the mass-based fit are shown. Values and uncertainties for estimates from the hierarchical model are reported in Appendix S2: Tables S1, S2.

uncertainty, but this benefit was primarily detectable only at very small sample sizes.

\section{Discussion}

\section{Scale dependence of the leaf economic spectrum}

Our first objective was to investigate the extent to which the global relationships defined by the leaf economic spectrum, namely, positive relationships among SLA, $\mathrm{N}_{\text {mass }}, \mathrm{P}_{\text {mass }}$, and $R_{\mathrm{d} \text {,mass }}$ and negative relationships of all these traits with leaf lifespan (Wright et al. 2004, Shipley et al. 2006, Reich 2014, Díaz et al. 2015), hold within and across PFTs. Our results suggest that, among the seven traits we investigated, there are three levels of "robustness" for leaf economic relationships. The top tier of leaf economic relationships involves morphological and biochemical traits, SLA, N, 


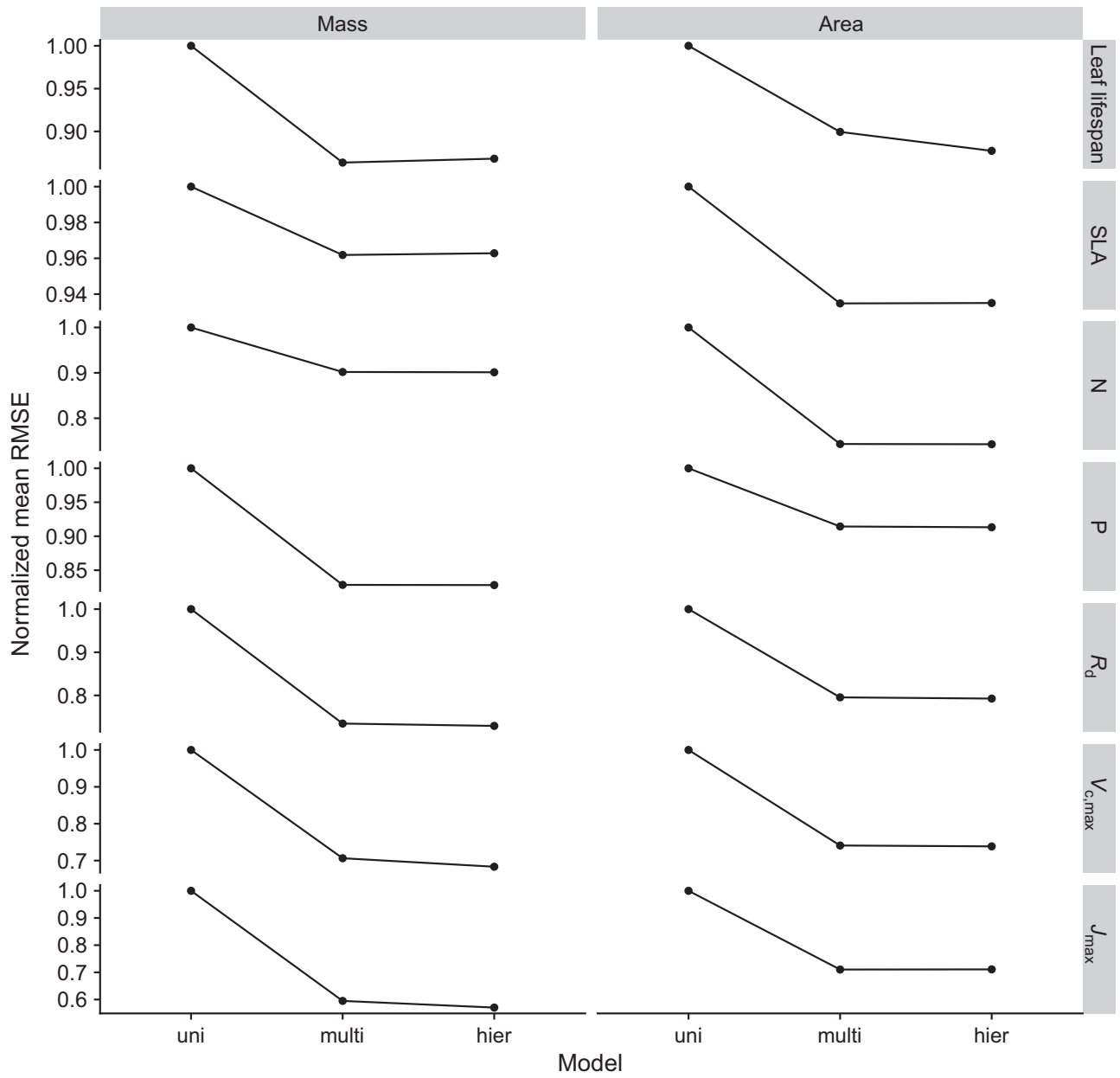

FIG. 3. Normalized mean root mean square error (RMSE) estimates from 20 -fold cross-validation, by model and trait. Normalization is such that the highest RMSE for a given trait-model combination is 1. Model abbreviations are as follows: "uni" is the univariate model fit separately to each PFT; "multi" is the multivariate model fit separately to each PFT; and "hier" is the hierarchical model.

and $\mathrm{P}$, which had covariance patterns consistent with the leaf economic spectrum both across PFTs and within all PFTs. The second tier involves metabolic traits, $R_{\mathrm{d}}$, $V_{\mathrm{c}, \text { mass, }}$ and $J_{\max }$, which were generally consistent with the leaf economic spectrum, but with a weaker relationship across PFTs and with notable deviations within specific PFTs. The third tier involves leaf lifespan, which had a relatively strong leaf economic spectrum signal across PFTs and within a majority of PFTs, but which showed systematic deviations from the leaf economic spectrum within many PFTs.

The consistent direction of relationships among SLA, N, and P (by mass and area) across and within all PFTs suggests that they are driven by processes that are more-or-less universal (Fig. 1). The consistent positive relationship between $\mathrm{N}$ and $\mathrm{P}$ (by mass or area) reflects the tight stoichiometric link between these two nutrients, and suggests that the variations in nutrient supply that would drive changes in the $\mathrm{N}: \mathrm{P}$ ratio are larger within-PFTs than across (Elser et al. 2010). Meanwhile, the consistent positive relationships of SLA with mass-

normalized $\mathrm{N}$ and $\mathrm{P}$ reflects the fact that increases in leaf mass per area (i.e., decreases in SLA) are driven primarily by increases in structural carbohydrates, which inevitably leads to a decline in nutrient mass fractions (Poorter et al. 2009). At the same time, the consistent negative relationships of SLA with areanormalized $\mathrm{N}$ and $\mathrm{P}$ reflect the role of these nutrients in structural proteins (Onoda et al. 2017). It should be noted that, although the direction of SLA- $\mathrm{N}_{\text {mass }}$ and SLA-P $P_{\text {mass }}$ relationships was consistent, the magnitude of their slopes showed non-trivial variation, particularly on a mass basis.

The less robust leaf economic spectrum signal in metabolic traits (Fig. 1) is likely a combination of two factors: more plasticity in plant metabolism relative to morphological and biochemical traits, and much smaller 


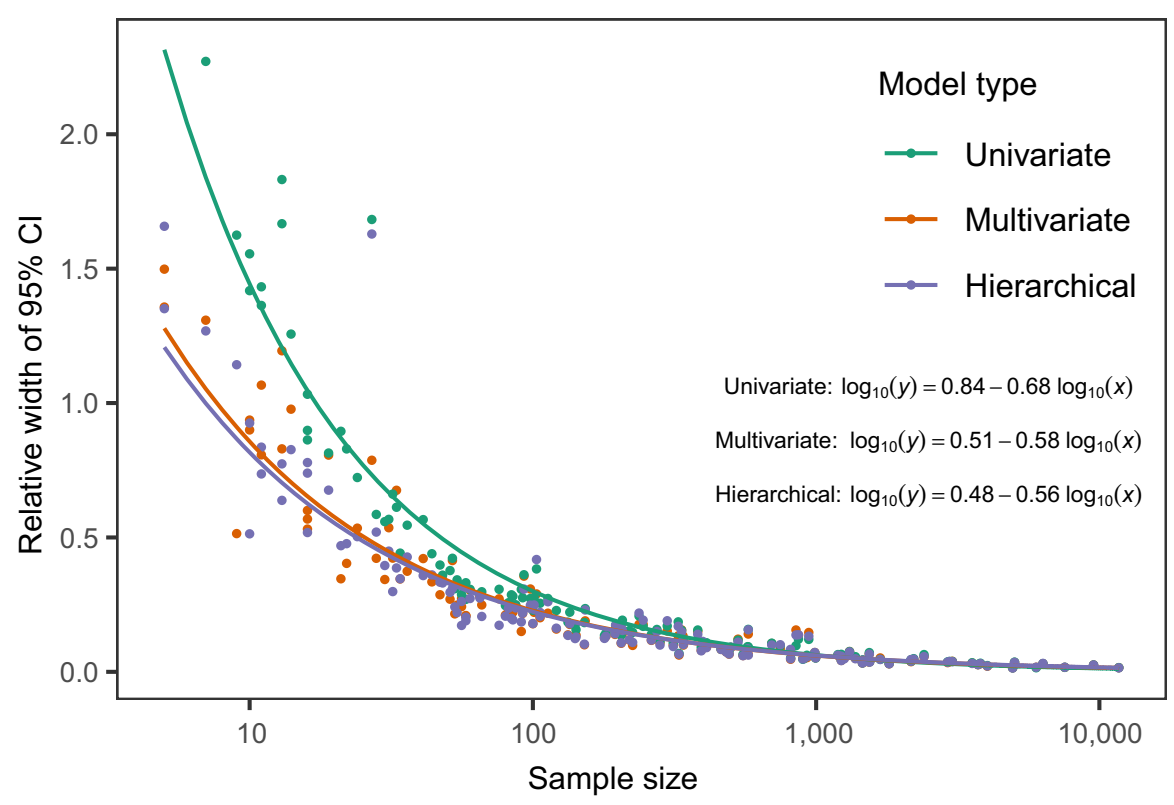

FIG. 4. Relative uncertainty in plant functional type-level trait estimates as a function of sample size for each model type. Lines represent linear models $\left(\log (y)=b_{0}+b_{1} \log (x)\right)$ fit independently for each model type. In general, differences in estimate uncertainty between the univariate and multivariate models were minimal at large sample sizes but increasingly important at low sample sizes. However, differences in estimate uncertainty between the multivariate and hierarchical models were consistently negligible.

sample sizes for confidently estimating relationships. Plasticity in plant metabolic traits independent of the leaf economic spectrum is well documented. For example, Kattge et al. (2009) showed that across-PFT variation in $V_{\mathrm{c} \text {,max,area }}$ was driven by differences in photosynthetic $\mathrm{N}$ use efficiency while variation within PFTs was driven by differences in $\mathrm{N}$ content, and that $\mathrm{N}_{\text {area }}-V_{\mathrm{c} \text {,max,area }}$ relationships within PFTs were variable. More generally, there is substantial variability across PFTs in how leaf $\mathrm{N}$ is allocated to photosynthesis (Ghimire et al. 2017) and across leaf biochemical consituents more generally (Onoda et al. 2011). The scale dependence we observed in $V_{\mathrm{c}, \max }-J_{\max }$ relationship, namely, that its slope was consistent within PFTs, but very weak across PFTs, may be a reflection of strong variation in growth irradiance and temperature across biomes, which have been shown to alter the $J_{\max } / V_{\mathrm{c}, \max }$ ratio (Hikosaka 2005, Hikosaka et al. 2005, Xiang et al. 2013). An important limitation to these results is the relative scarcity of metabolic trait measurements, especially for highlatitude PFTs (Table 1; Appendix S2: Table S4). More simultaenous observations of metabolic traits and other leaf economic traits on the same leaf samples are needed to better understand how much these are actual ecological patterns vs. just artifacts of sampling bias.

The fact that trait relationships involving leaf lifespan showed the most scale dependence and within-PFT variability (Fig. 1) is not particularly surprising considering that leaf habit (deciduous vs. evergreen), the largest driver of global variability in leaf lifespan, is a part of the PFT definition. As noted by Wright et al. (2004) in their original presentation of the leaf economic spectrum, specific leaf area and leaf lifespan were decoupled in deciduous species, largely because of these specues' relatively small variation in leaf lifespan. The very inconsistent direction of relationships of area-normalized traits with leaf lifespan is also consistent with the results of Wright et al. (2004). The systematic differences in the leaf lifespan-SLA relationship with biome we observed among deciduous PFTs can be interpreted in terms of withinPFT climate variability. Specifically, for deciduous species, leaf lifespan is primarily driven by the length of the local growing season, which generally decreases with annual mean temperature, whereas the larger variability in leaf lifespan of evergreen species is less sensitive (or even inversely related) to changes in climate (Appendix S2: Fig. S2). Ultimately, this suggests that leaf economic relationships related to leaf lifespan are dominated by acrossPFT differences, particularly those between deciduous and evergreen PFTs, while factors driving variability in leaf lifespan within PFTs are more complex and idiosyncratic (Reich et al. 2014, Wu et al. 2016a).

\section{Covariance as constraint}

The second objective of this paper was to investigate the information content of trait covariance; i.e., how much more can we learn about specific traits based on their relationships with other traits? We show that accounting for covariance both improved the accuracy of trait imputation (Fig. 3) and reduced posterior predictive uncertainty around PFT-level trait means, 
particularly for undersampled trait-PFT combinations (Fig. 4; Appendix S2: Fig. S1). Moreover, accounting for covariance occasionally resulted in small but statistically significant differences in the position of trait mean estimates even for well-sampled PFT-trait combinations (e.g., $\mathrm{N}_{\text {mass }}$ for temperate broadleaved deciduous trees, Appendix S2: Fig. S1). This result echoes Díaz et al. (2015) in demonstrating the importance of studying the multivariate trait space rather than individual traits. Significant differences between univariate and multivariate estimates of trait means suggest that sampling of these traits in TRY is not representative (Table 1; Appendix S2: Section S2; see also Kattge et al. 2011). These differences also indicate that parameter estimates based on univariate trait data (LeBauer et al. 2013, Dietze et al. 2014, Butler et al. 2017) may not only overestimate uncertainty, but may also be systematically biased. Although some traits in our analysis $\left(R_{\mathrm{d}}, V_{\mathrm{c}, \max }\right.$, and $\left.J_{\max }\right)$ had too few observations to estimate covariance patterns for some PFTs with much statistical power, we show that leveraging covariance increases the effective sample size of all traits. This means that field and remote sensing studies that estimate only certain traits (like SLA and $\mathrm{N}_{\text {mass }}$ ) may be able to use trait correlations to provide constraint on traits they do not directly observe (such as $\mathrm{P}_{\text {mass }}$ and $R_{\mathrm{d} \text {,mass; }}$; Serbin et al. 2014, Musavi et al. 2015, Singh et al. 2015, Lepine et al. 2016). As such, future observational campaigns should consider trait covariance when deciding which traits to measure.

The additional benefit of hierarchical multivariate modeling in our study was limited, due to a combination of the low number of points used to estimate across-PFT covariance, the weak slopes of those relationships, and the usually consistent direction of pairwise slopes within and across PFTs. Therefore, for parameterizing the current generation of ecosystem models using well-sampled traits, simple multivariate models fit independently to each PFT may be sufficient and the additional conceptual challenges and computational overhead of hierarchical modeling are not required. However, for modeling larger numbers of PFTs, the benefits of hierarchical modeling may accumulate (Clark 2004, Dietze et al. 2008, Cressie et al. 2009, Webb et al. 2010), particularly in situations where within- and across-group covariance patterns differ. Future work should use similar methods, potentially in combination with additional information from phylogenetic or taxonomic similarity (Symonds and Blomberg 2014), to explore the extent to which leaf economic relationships hold within vs. across other groups, such as taxonomic levels (species, genus, family, clade), successional stages, or spatial domains.

This raises the question: What is the "best" way to represent plant functional diversity in the next generation of terrestrial ecosystem models? The current PFTs are products of an era in which computational power was more limited and data on functional diversity were relatively scarce (Prentice et al. 1992, Box 1995, Woodward and Cramer 1996); this study, among others, points to their limitations. This PFT structure is, however, not immutable. One alternative would be to explicitly account for systematic differences in trait values between regions with similar climates (Butler et al. 2017). A second alternative is to further disaggregate PFTs based on successional stage, shade tolerance, or similar ecological characteristic (Hickler et al. 2011, Longo et al. 2019). A third is to allow PFTs to emerge from the data by applying classification and clustering techniques to functional trait observations (Boulangeat et al. 2012). Finally, one could eschew PFTs in favor of modeling individual species (Post and Pastor 1996, Weng et al. 2015), or even abandon discrete categories altogether and model the continuous trait space (Scheiter et al. 2013). Our methods for quantifying trait covariance would benefit any or all of these approaches.

More generally, we foresee tremendous potential for multivariate and hierarchical modeling to elucidate the relationship between traits and organismal and ecosystem function. A natural next step would be to apply the same approach to traits whose relationship to the leaf economic spectrum is less clear. One example is hydraulic traits: While stem and leaf hydraulic traits are correlated (Bartlett et al. 2016), the scaling between hydraulic and leaf economic traits is poorly understood (Reich 2014, Li et al. 2015). Similarly, reexamining the relationships defining wood (Chave et al. 2009, Baraloto et al. 2010, Fortunel et al. 2012) and root (Kramer-Walter et al. 2016, Valverde-Barrantes and Blackwood 2016) economic spectra, as well as their relationship to the foliar traits, would provide useful information on scaledependence of plant growth and allocation strategies. The difficulty of measuring hydraulic and other nonfoliar traits (Jansen et al. 2015) further increases the value of any technique that can fully leverage the information they provide. Ultimately, multivariate and hierarchical modeling may reveal functional trade-offs that are mutually confounding at different scales, thereby enhancing our understanding of processes driving functional diversity.

\section{ACKNOWLeDGments}

This project was supported by NASA grant NNX14AH65G and NSF grants 1261582, 1458021, and 1655095, as well as the TRY initiative on plant traits (http://www.try-db.org). The TRY initiative and database is hosted, developed, and maintained by J. Kattge and G. Boenisch (Max Planck Institute for Biogeochemistry, Jena, Germany). TRY is currently supported by DIVERSITAS/Future Earth and the German Centre for Integrative Biodiversity Research (iDiv) Halle-Jena-Leipzig. The authors would also like to thank Ben Bond-Lamberty, Christine Rollinson, Istem Fer, and Colin Averill for their valuable feedback on early drafts of this manuscript. A. N. Shiklomanov wrote the manuscript and implemented the analysis. A. N. Shiklomanov and E. M. Cowdery designed the analysis and figures. M. C. Dietze conceived the original idea for the manuscript, guided its development, and provided financial support. M. Bahn, S. Jansen, K. Kramer, Ü. Niinemets, and N. A. Soudzilovskaia provided extensive feedback on multiple drafts of the 
manuscript, and contributed data. C. Byun and Y. Onoda contributed data.

\section{Literature Cited}

Albert, C. H., W. Thuiller, N. G. Yoccoz, R. Douzet, S. Aubert, and S. Lavorel. 2010a. A multi-trait approach reveals the structure and the relative importance of intra- vs. interspecific variability in plant traits. Functional Ecology 24:1192-1201.

Albert, C. H., W. Thuiller, N. G. Yoccoz, A. Soudant, F. Boucher, P. Saccone, and S. Lavorel. 2010b. Intraspecific functional variability: extent, structure and sources of variation. Journal of Ecology 98:604-613.

Asner, G. P., R. E. Martin, R. Tupayachi, C. B. Anderson, F. Sinca, L. Carranza-Jimenez, and P. Martinez. 2014. Amazonian functional diversity from forest canopy chemical assembly. Proceedings of the National Academy of Sciences USA 111:5604-5609.

Atkin, O. K., et al. 2015. Global variability in leaf respiration in relation to climate, plant functional types and leaf traits. New Phytologist 206:614-636.

Bakker, M. A., G. Carreño-Rocabado, and L. Poorter. 2010. Leaf economics traits predict litter decomposition of tropical plants and differ among land use types. Functional Ecology 25:473-483.

Baraloto, C., C. E. T. Paine, L. Poorter, J. Beauchene, D. Bonal, A.-M. Domenach, B. Hérault, S. Patiño, J.-C. Roggy, and J. Chave. 2010. Decoupled leaf and stem economics in rain forest trees. Ecology Letters 13:1338-1347.

Bartlett, M. K., T. Klein, S. Jansen, B. Choat, and L. Sack 2016. The correlations and sequence of plant stomatal, hydraulic, and wilting responses to drought. Proceedings of the National Academy of Sciences USA 113:13098-13103.

Boulangeat, I., P. Philippe, S. Abdulhak, R. Douzet, L. Garraud, S. Lavergne, S. Lavorel, J. Van Es, P. Vittoz, and W. Thuiller. 2012. Improving plant functional groups for dynamic models of biodiversity: at the crossroads between functional and community ecology. Global Change Biology 18:3464-3475.

Box, E. O. 1995. Factors determining distributions of tree species and plant functional types. Vegetatio 121:101-116.

Burns, K. C. 2004. Patterns in specific leaf area and the structure of a temperate heath community. Diversity and Distributions 10:105-112.

Butler, E. E., et al. 2017. Mapping local and global variability in plant trait distributions. Proceedings of the National Academy of Sciences USA 114:E10937-E10946.

Cardinale, B. J., et al. 2012. Biodiversity loss and its impact on humanity. Nature 486:59-67.

Cavender-Bares, J., K. Kitajima, and F. A. Bazzaz. 2004. Multiple trait associations in relation to habitat differentiation among 17 Floridian oak species. Ecological Monographs 74:635-662.

Champely, S. 2018. pwr: basic functions for power analysis. R package version 1.2-2. https://github.com/heliosdrm/pwr/tree/ v1.2-2

Chave, J., D. Coomes, S. Jansen, S. L. Lewis, N. G. Swenson, and A. E. Zanne. 2009. Towards a worldwide wood economics spectrum. Ecology Letters 12:351-366.

Clark, J. S. 2004. Why environmental scientists are becoming Bayesians. Ecology Letters 8:2-14.

Clark, J. S. 2016. Why species tell more about traits than traits about species: predictive analysis. Ecology 97:1979-1993.

Clements, F. E. 1936. Nature and structure of the climax. Journal of Ecology 24:252.

Cornwell, W. K., and D. D. Ackerly. 2009. Community assembly and shifts in plant trait distributions across an environmental gradient in coastal California. Ecological Monographs 79:109-126.

Cornwell, W. K., W. D. Pearse, R. L. Dalrymple, and A. E. Zanne. 2019. What we (don't) know about global plant diversity. Ecography 42:1819-1831.

Cowles, H. C. 1899. The ecological relations of the vegetation on the sand dunes of Lake Michigan. Part I - Geographical relations of the dune floras. Botanical Gazette 27:95-117.

Cressie, N., C. A. Calder, J. S. Clark, J. M. V. Hoef, and C. K. Wikle. 2009. Accounting for uncertainty in ecological analysis: the strengths and limitations of hierarchical statistical modeling. Ecological Applications 19:553-570.

Diaz, S., et al. 2004. The plant traits that drive ecosystems: evidence from three continents. Journal of Vegetation Science 15:295-304

Díaz, S., et al. 2015. The global spectrum of plant form and function. Nature 529:167-171.

Dietze, M. C., D. S. LeBauer, and R. Kooper. 2013. On improving the communication between models and data. Plant, Cell \& Environment 36:1575-1585.

Dietze, M. C., M. S. Wolosin, and J. S. Clark. 2008. Capturing diversity and interspecific variability in allometries: a hierarchical approach. Forest Ecology and Management 256:19391948.

Dietze, M. C., et al. 2014. A quantitative assessment of a terrestrial biosphere model's data needs across North American biomes. Journal of Geophysical Research: Biogeosciences 119:286-300.

Edwards, E. J., D. S. Chatelet, L. Sack, and M. J. Donoghue. 2014. Leaf life span and the leaf economic spectrum in the context of whole plant architecture. Journal of Ecology 102:328-336.

Elser, J. J., W. F. Fagan, A. J. Kerkhoff, N. G. Swenson, and B. J. Enquist. 2010. Biological stoichiometry of plant production: metabolism, scaling and ecological response to global change. New Phytologist 186:593-608.

Feng, X., and M. Dietze. 2013. Scale dependence in the effects of leaf ecophysiological traits on photosynthesis: Bayesian parameterization of photosynthesis models. New Phytologist 200:1132-1144.

Fick, S. E., and R. J. Hijmans. 2017. Worldclim 2: New 1-km spatial resolution climate surfaces for global land areas. International Journal of Climatology 37:4302-4315.

Fortunel, C., P. V. A. Fine, and C. Baraloto. 2012. Leaf, stem and root tissue strategies across 758 neotropical tree species. Functional Ecology 26:1153-1161.

Friedlingstein, P., et al. 2006. Climate-carbon cycle feedback analysis: results from the C4MIP model intercomparison. Journal of Climate 19:3337-3353.

Friedlingstein, P., M. Meinshausen, V. K. Arora, C. D. Jones, A. Anav, S. K. Liddicoat, and R. Knutti. 2014. Uncertainties in CMIP5 climate projections due to carbon cycle feedbacks. Journal of Climate 27:511-526.

Gelman, A., J. Carlin, H. Stern, and D. Rubin. 2003. Bayesian data analysis. Chapman \& Hall/CRC Texts in Statistical Science. Chapman \& Hall/CRC, Boca Raton, Florida, USA.

Gelman, A., and D. B. Rubin. 1992. Inference from iterative simulation using multiple sequences. Statistical Science 7:457-472.

Ghimire, B., W. J. Riley, C. D. Koven, J. Kattge, A. Rogers, P. B. Reich, and I. J. Wright. 2017. A global trait-based approach to estimate leaf nitrogen functional allocation from observations. Ecological Applications 27:1421-1434.

Graham, J. W. 2009. Missing data analysis: making it work in the real world. Annual Review of Psychology 60:549-576. 
Grime, J. P. 1998. Benefits of plant diversity to ecosystems: immediate, filter and founder effects. Journal of Ecology 86:902-910

Grime, J. P., and S. Pierce. 2012. The evolutionary strategies that shape ecosystems. John Wiley \& Sons Ltd., Hoboken, New Jersey, USA.

Grubb, P., T. Marañón, F. Pugnaire, and L. Sack. 2015. Relationships between specific leaf area and leaf composition in succulent and non-succulent species of contrasting semidesert communities in south-eastern Spain. Journal of Arid Environments 118:69-83.

Haario, H., E. Saksman, and J. Tamminen. 2001. An adaptive Metropolis algorithm. Bernoulli 7:223.

Hickler, T., et al. 2011. Projecting the future distribution of European potential natural vegetation zones with a generalized, tree species-based dynamic vegetation model. Global Ecology and Biogeography 21:50-63.

Hikosaka, K. 2005. Nitrogen partitioning in the photosynthetic apparatus of Plantago asiatica leaves grown under different temperature and light conditions: similarities and differences between temperature and light acclimation. Plant and Cell Physiology 46:1283-1290.

Hikosaka, K., K. Ishikawa, A. Borjigidai, O. Muller, and Y. Onoda. 2005. Temperature acclimation of photosynthesis: mechanisms involved in the changes in temperature dependence of photosynthetic rate. Journal of Experimental Botany 57:291-302.

Hobbie, S. E. 2015. Plant species effects on nutrient cycling: revisiting litter feedbacks. Trends in Ecology \& Evolution 30:357-363.

Jansen, S., B. Schuldt, and B. Choat. 2015. Current controversies and challenges in applying plant hydraulic techniques. New Phytologist 205:961-964.

Kattge, J., and W. Knorr. 2007. Temperature acclimation in a biochemical model of photosynthesis: a reanalysis of data from 36 species. Plant, Cell \& Environment 30:1176-1190.

Kattge, J., W. Knorr, T. Raddatz, and C. Wirth. 2009. Quantifying photosynthetic capacity and its relationship to leaf nitrogen content for global-scale terrestrial biosphere models. Global Change Biology 15:976-991.

Kattge, J., et al. 2011. TRY - a global database of plant traits. Global Change Biology 17:2905-2935.

Kichenin, E., D. A. Wardle, D. A. Peltzer, C. W. Morse, and G. T. Freschet. 2013. Contrasting effects of plant inter- and intraspecific variation on community-level trait measures along an environmental gradient. Functional Ecology 27:1254-1261.

Kleyer, M., and V. Minden. 2015. Why functional ecology should consider all plant organs: an allocation-based perspective. Basic and Applied Ecology 16:1-9.

Kramer-Walter, K. R., P. J. Bellingham, T. R. Millar, R. D. Smissen, S. J. Richardson, and D. C. Laughlin. 2016. Root traits are multidimensional: specific root length is independent from root tissue density and the plant economic spectrum. Journal of Ecology 104:1299-1310.

Lavorel, S., S. McIntyre, J. Landsberg, and T. Forbes. 1997. Plant functional classifications: from general groups to specific groups based on response to disturbance. Trends in Ecology \& Evolution 12:474-478.

LeBauer, D. S., D. Wang, K. T. Richter, C. C. Davidson, and M. C. Dietze. 2013. Facilitating feedbacks between field measurements and ecosystem models. Ecological Monographs 83:133-154.

Lepine, L. C., S. V. Ollinger, A. P. Ouimette, and M. E. Martin. 2016. Examining spectral reflectance features related to foliar nitrogen in forests: implications for broad-scale nitrogen mapping. Remote Sensing of Environment 173:174-186.
Li, L., M. L. McCormack, C. Ma, D. Kong, Q. Zhang, X. Chen, H. Zeng, Ü. Niinemets, and D. Guo. 2015. Leaf economics and hydraulic traits are decoupled in five species-rich tropical-subtropical forests. Ecology Letters 18:899-906.

Lloyd, J., K. Bloomfield, T. F. Domingues, and G. D. Farquhar. 2013. Photosynthetically relevant foliar traits correlating better on a mass vs an area basis: of ecophysiological relevance or just a case of mathematical imperatives and statistical quicksand? New Phytologist 199:311-321.

Longo, M., et al. 2019. The biophysics, ecology, and biogeochemistry of functionally diverse, vertically- and horizontallyheterogeneous ecosystems: the Ecosystem Demography model, version 2.2 - part 1: model description. Geoscientific Model Development Discussions 12:4309-4346.

Messier, J., M. J. Lechowicz, B. J. McGill, C. Violle, and B. J. Enquist. 2017a. Interspecific integration of trait dimensions at local scales: the plant phenotype as an integrated network. Journal of Ecology 105:1775-1790.

Messier, J., B. J. McGill, B. J. Enquist, and M. J. Lechowicz. 2017b. Trait variation and integration across scales: Is the leaf economic spectrum present at local scales? Ecography 40:685-697.

Messier, J., B. J. McGill, and M. J. Lechowicz. 2010. How do traits vary across ecological scales? A case for trait-based ecology. Ecology Letters 13:838-848.

Musavi, T., et al. 2015. The imprint of plants on ecosystem functioning: a data-driven approach. International Journal of Applied Earth Observation and Geoinformation 43:119-131.

Naeem, S., and J. P. Wright. 2003. Disentangling biodiversity effects on ecosystem functioning: deriving solutions to a seemingly insurmountable problem. Ecology Letters 6:567579.

Neal, R. M. 2011. MCMC using Hamiltonian dynamics. Pages 113-163 in S. Brooks, A. Gelman, G. L. Jones, and X-L. Meng, editors. Handbook of Markov Chain Monte Carlo. Chapman \& Hall/CRC, Boca Raton, Florida, USA.

Niinemets, Ü. 2016. Within-canopy variations in functional leaf traits: structural, chemical and ecological controls and diversity of responses. Pages 101-141 in K. Hikosaka, Ü. Niinemets, and N. Anten, editors. Canopy photosynthesis: from basics to applications advances in photosynthesis and respiration (including bioenergy and related process), vol. 42 . Springer, Dordrecht, The Netherlands.

Oleson, K. W., et al. 2013. Technical description of version 4.5 of the Community Land Model (CLM). Technical Report NCAR/TN-503+STR, NCAR Earth System Laboratory Climate and Global Dynamics Division. National Center for Atmospheric Research, Boulder, Colorado, USA.

Onoda, Y., et al. 2011. Global patterns of leaf mechanical properties. Ecology Letters 14:301-312.

Onoda, Y., I. J. Wright, J. R. Evans, K. Hikosaka, K. Kitajima, Ü. Niinemets, H. Poorter, T. Tosens, and M. Westoby. 2017. Physiological and structural tradeoffs underlying the leaf economics spectrum. New Phytologist 214:1447-1463.

Ordoñez, J. C., P. M. van Bodegom, J.-P. M. Witte, I. J. Wright, P. B. Reich, and R. Aerts. 2009. A global study of relationships between leaf traits, climate and soil measures of nutrient fertility. Global Ecology and Biogeography 18:137-149.

Osnas, J. L. D., J. W. Lichstein, P. B. Reich, and S. W. Pacala. 2013. Global leaf trait relationships: mass, area, and the leaf economics spectrum. Science 340:741-744.

Poorter, H., and M.-L. Navas. 2003. Plant growth and competition at elevated $\mathrm{CO}_{2}$ : on winners, losers and functional groups. New Phytologist 157:175-198.

Poorter, H., Ü. Niinemets, L. Poorter, I. J. Wright, and R. Villar. 2009. Causes and consequences of variation in leaf mass 
per area (LMA): a meta-analysis. New Phytologist 182:565588.

Post, W. M., and J. Pastor. 1996. Linkages: an individual-based forest ecosystem model. Climatic Change 34:253-261.

Prentice, I. C., W. Cramer, S. P. Harrison, R. Leemans, R. A. Monserud, and A. M. Solomon. 1992. Special paper: a global biome model based on plant physiology and dominance, soil properties and climate. Journal of Biogeography 19:117.

R Core Team. 2019. R: a language and environment for statistical computing. R Foundation for Statistical Computing, Vienna, Austria. www.R-project.org

Reich, P. B. 2014. The world-wide 'fast-slow' plant economics spectrum: a traits manifesto. Journal of Ecology 102:275301.

Reich, P. B., R. L. Rich, X. Lu, Y.-P. Wang, and J. Oleksyn. 2014. Biogeographic variation in evergreen conifer needle longevity and impacts on boreal forest carbon cycle projections. Proceedings of the National Academy of Sciences USA 111:13703-13708.

Rosado, B. H. P., and E. A. de Mattos. 2017. On the relative importance of CSR ecological strategies and integrative traits to explain species dominance at local scales. Functional Ecology 31:1969-1974.

Sakschewski, B., W. von Bloh, A. Boit, A. Rammig, J. Kattge, L. Poorter, J. Peñuelas, and K. Thonicke. 2015. Leaf and stem economics spectra drive diversity of functional plant traits in a dynamic global vegetation model. Global Change Biology 21:2711-2725.

Scheiter, S., L. Langan, and S. I. Higgins. 2013. Next-generation dynamic global vegetation models: learning from community ecology. New Phytologist 198:957-969.

Serbin, S. P., A. Singh, B. E. McNeil, C. C. Kingdon, and P. A. Townsend. 2014. Spectroscopic determination of leaf morphological and biochemical traits for northern temperate and boreal tree species. Ecological Applications 24:1651-1669.

Shaw, R. G., and J. R. Etterson. 2012. Rapid climate change and the rate of adaptation: insight from experimental quantitative genetics. New Phytologist 195:752-765.

Shipley, B., M. J. Lechowicz, I. Wright, and P. B. Reich. 2006. Fundamental trade-offs generating the worldwide leaf economics spectrum. Ecology 87:535-541.

Shipley, B., D. Vile, E. Garnier, I. J. Wright, and H. Poorter. 2005. Functional linkages between leaf traits and net photosynthetic rate: reconciling empirical and mechanistic models. Functional Ecology 19:602-615.

Singh, A., S. P. Serbin, B. E. McNeil, C. C. Kingdon, and P. A. Townsend. 2015. Imaging spectroscopy algorithms for mapping canopy foliar chemical and morphological traits and their uncertainties. Ecological Applications 25:2180-2197.

Sitch, S., et al. 2008. Evaluation of the terrestrial carbon cycle, future plant geography and climate-carbon cycle feedbacks using five Dynamic Global Vegetation Models (DGVMs). Global Change Biology 14:2015-2039.

Swenson, N. G. 2013. Phylogenetic imputation of plant functional trait databases. Ecography 37:105-110.

Symonds, M. R. E., and S. P. Blomberg. 2014. A primer on phylogenetic generalised least squares. Page 105-130 in L. Garamszegi, editor. Modern phylogenetic comparative methods and their application in evolutionary biology. Springer, Berlin, Heidelberg.

ter Braak, C. J. F., and J. A. Vrugt. 2008. Differential evolution Markov Chain with snooker updater and fewer chains. Statistics and Computing 18:435-446.

Valverde-Barrantes, O. J. and C. B. Blackwood. 2016. Root traits are multidimensional: specific root length is independent from root tissue density and the plant economic spectrum: Commentary on Kramer-Walteret et al. (2016). Journal of Ecology 104:1311-1313.

Van Bodegom, P. M., J. C. Douma, J. P. M. Witte, J. C. Ordoñez, R. P. Bartholomeus, and R. Aerts. 2011. Going beyond limitations of plant functional types when predicting global ecosystem-atmosphere fluxes: exploring the merits of traits-based approaches. Global Ecology and Biogeography 21:625-636.

Verheijen, L. M., R. Aerts, V. Brovkin, J. Cavender-Bares, J. H. C. Cornelissen, J. Kattge, and P. M. van Bodegom. 2015. Inclusion of ecologically based trait variation in plant functional types reduces the projected land carbon sink in an earth system model. Global Change Biology 21:3074-3086.

Violle, C., M.-L. Navas, D. Vile, E. Kazakou, C. Fortunel, I. Hummel, and E. Garnier. 2007. Let the concept of trait be functional! Oikos 116:882-892.

Webb, C. T., J. A. Hoeting, G. M. Ames, M. I. Pyne, and N. LeRoy Poff. 2010. A structured and dynamic framework to advance traits-based theory and prediction in ecology. Ecology Letters 13:267-283.

Weng, E. S., S. Malyshev, J. W. Lichstein, C. E. Farrior, R. Dybzinski, T. Zhang, E. Shevliakova, and S. W. Pacala. 2015. Scaling from individual trees to forests in an Earth System Modeling framework using a mathematically tractable model of height-structured competition. Biogeosciences 12:26552694.

Westoby, M., D. S. Falster, A. T. Moles, P. A. Vesk, and I. J. Wright. 2002. Plant ecological strategies: some leading dimensions of variation between species. Annual Review of Ecology and Systematics 33:125-159.

White, I. R., P. Royston, and A. M. Wood. 2010. Multiple imputation using chained equations: issues and guidance for practice. Statistics in Medicine 30:377-399.

Wigley, B. J., J. A. Slingsby, S. Díaz, W. J. Bond, H. Fritz, and C. Coetsee. 2016. Leaf traits of African woody savanna species across climate and soil fertility gradients: evidence for conservative versus acquisitive resource-use strategies. Journal of Ecology 104:1357-1369.

Woodward, F. I., and W. Cramer. 1996. Plant functional types and climatic change: introduction. Journal of Vegetation Science 7:306-308

Wright, J. P., and A. Sutton-Grier. 2012. Does the leaf economic spectrum hold within local species pools across varying environmental conditions? Functional Ecology 26:1390-1398.

Wright, I. J., et al. 2004. The worldwide leaf economics spectrum. Nature 428:821-827.

Wright, I. J., et al. 2005. Modulation of leaf economic traits and trait relationships by climate. Global Ecology and Biogeography $14: 411-421$.

Wu, J., et al. 2016a. Leaf development and demography explain photosynthetic seasonality in Amazon evergreen forests. Science 351:972-976.

Wu, J., et al. 2016b. Convergence in relationships between leaf traits, spectra and age across diverse canopy environments and two contrasting tropical forests. New Phytologist 214:1033-1048.

Wullschleger, S. D., H. E. Epstein, E. O. Box, E. S. Euskirchen, S. Goswami, C. M. Iversen, J. Kattge, R. J. Norby, P. M. van Bodegom, and X. Xu. 2014. Plant functional types in earth system models: past experiences and future directions for application of dynamic vegetation models in high-latitude ecosystems. Annals of Botany 114:1-16

Xiang, S., P. B. Reich, S. Sun, and O. K. Atkin. 2013. Contrasting leaf trait scaling relationships in tropical and temperate wet forest species. Functional Ecology 27:522-534. 
SUPPORTING INFORMATION

Additional supporting information may be found online at: http://onlinelibrary.wiley.com/doi/10.1002/eap.2064/full

Data Availability

$\mathrm{R}$ code and data are available from the Open Science Framework: https://osf.io/w8y73/. Trait data are archived in the TRY Plant Trait Database at http://www.try-db.org and can be retrieved by providing TRY data request ID \#1584. 